\title{
Evolución del Programa de Asistencia Psiquiátrica a Personas Sin Hogar con Enfermedad Mental Grave en el municipio de Bilbao
}

\section{Mariasun Garay}

Red de Salud Mental de Bizkaia, Osakidetza

$<$ mariaasuncion.garayarostegui@osakidetza.net>

\section{Ernesto Notario}

Sección de Inclusión y Urgencias Sociales, Ayuntamiento de Bilbao

\section{José María Duque}

Sección de Inclusión y Urgencias Sociales, Ayuntamiento de Bilbao

\section{Adela Olaskoaga}

Dirección Territorial de Bizkaia, Consejería de Sanidad y Consumo, Gobierno Vasco

\section{José J. Uriarte}

Red de Salud Mental de Bizkaia, Osakidetza

Bertan aurkezten da sei urteko epe luzean egindako Buruko Gaixotasun Larriak dituzten Etxerik Gabeko Pertsonen Arreta Psikiatrikorako Programa. Bilboko Udalak, Bizkaiko Buru Osasuneko Sareak, udaleko Gizarte Ekintza Sailak eta Eusko Jaurlaritzaren Osasun eta Kontsumo Sailak elkarrekin garatutako programa izan da hori. Bere xedea da kolektibo horretako pertsonen buruko gaixotasun larriak detektatzea eta arreta ematea, izan ere, ez baitira iristen arreta psikiatrikoa jasotzera, eta, gainera, normalizatutako buru-osasun zerbitzuetara jotzeko zailtasun ugari izaten dute. Biztanle horien ezaugarriak azaltzen dira, zerbitzuak hasieratik artatutako 90 kasuak oinarri harturik, eta giltzarri hartutako metodologia eta berrikuntza hauek iradokitzen dituzte: asertibotasunaren eredura eraldatzea artatzearen eredu hori eta alor soziosanitarioko koordinazioa.

\section{HITZ-GAKOAK:}

buruko gaixotasun larria, etxegabeko pertsonak, zerbitzuen koordinazioa, koordinazio soziosanitarioa, programen ebaluazioa.
Se presenta un estudio longitudinal de seis años de duración del Programa de Atención Psiquiátrica a Personas Sin Hogar con Enfermedad Mental Grave, desarrollado conjuntamente en Bilbao por la Red de Salud Mental de Bizkaia, el Área de Acción Social del Ayuntamiento, y el Departamento de Sanidad y Consumo del Gobierno Vasco. Está destinado a detectar y atender la enfermedad mental grave en este colectivo, que, por su problemática específica, no accede a la asistencia psiquiátrica y presenta gran dificultad para vincularse con los servicios de salud mental normalizados. Se plantean las características de esta población en los más de 90 casos atendidos desde su inicio y lo que consideramos que son las claves metodológicas e innovadoras de este abordaje: el cambio asistencial estructural de tipo asertivo y la coordinación en el ámbito sociosanitario.

\section{Palabras Clave:}

enfermedad mental grave, personas sin hogar, coordinación de servicios, coordinación sociosanitaria, evaluación de programas. 


\section{Objetivo y justificación}

Resulta obvio decir que la situación de las personas sin hogar (PSH en adelante) es considerada como una de las formas más extremas de pobreza y exclusión social que se puedan dar en nuestra sociedad. Sin embargo, el abordaje de dicha problemática no ha tomado hasta relativamente hace unos pocos años un papel relevante en el conjunto de las políticas sociales. Por fortuna, cada vez suscita mayor interés tanto en el ámbito europeo como en el estatal, y este avance también ha tenido su repercusión a nivel local en la implantación de estrategias destinadas a combatir este fenómeno, complejo, difícil y de importante impacto. El proyecto que presentamos en este artículo es uno de los frutos de estas líneas de actuación.

La memoria realizada por el Área de Acción Social del Ayuntamiento de Bilbao en 2011 recoge que durante ese año recibieron prestaciones elementales (alojamiento, comida y vestido) 4.048 personas, y que 317 fueron atendidas específicamente por los equipos de calle. El análisis y abordaje de este colectivo ha permitido ampliar el conocimiento que tenemos sobre él y apreciar sus particularidades. El estudio Perfil de necesidades de las personas sin hogar de Bizkaia (Aierdi et al., 2009) observa que uno de los rasgos que ha ido aumentando con el paso de los años es la presencia de problemas de salud mental o de patología dual. Siguiendo los datos aportados por Eustat-Instituto Vasco de Estadística (2007) y Vázquez (2010), aproximadamente un $25 \%$ de esta población padece algún tipo de patología mental.

Los primeros estudios sistemáticos para conocer la situación de salud mental de las personas sin hogar realizados por el Instituto de Salud Mental de los Estados Unidos (NIMH) a finales de la década de los ochenta encontraron que las tasas de enfermedad mental grave, como la psicosis o la depresión severa, no eran tan altas como se esperaba y que el consumo de drogas tampoco parecía explicar por sí mismo el problema, a excepción del consumo de alcohol, que era un problema grave para cerca de la mitad de las personas sin hogar.

En Europa no fue hasta principios de los noventa cuando se llevaron a cabo los primeros estudios en París, Londres y Madrid (Muñoz, 2010). Un reciente metanálisis sobre 29 trabajos resume los resultados de todos estos estudios epidemiológicos de salud mental en PSH (Fazel et al., 2008). La muestra completa suma 5.684 personas sin hogar de distintos países occidentales, analizadas en el periodo comprendido entre 1979 y 2005 . Los resultados son dispares y heterogéneos, y muestran bastante dispersión, pero hablando en términos generales sobre la prevalencia media de las dolencias más frecuentes respecto al colectivo total, la dependencia del alcohol era del $37,9 \%$; la dependencia de otras sustancias, del 24,4\%; los trastornos psicóticos, del 12,7\%; la depresión mayor, del 11,4\%; y los trastornos de personalidad, del $23,1 \%$.
Desde el ámbito sanitario también se detectan una serie de insuficiencias en el abordaje de este colectivo. La inadaptación de los servicios de primera línea y la ausencia de equipos de referencia específicos saturan los recursos de alta intensidad, como las urgencias y las unidades hospitalarias, que genera un bucle de elevado coste personal y social.

Siendo sensibles a este marco epidemiológico y a las deficiencias estructurales de la atención sanitaria, se plantea el desarrollo del proyecto asistencial que exponemos. Su misión es ofrecer un programa de atención psiquiátrica a PSH afectadas por problemas de salud mental grave en el municipio de Bilbao. Es producto de un convenio de colaboración entre el Área de Acción Social del Ayuntamiento de Bilbao, la Dirección Territorial de Bizkaia de la Consejería de Sanidad y Consumo del Gobierno Vasco y la Red de Salud Mental de Bizkaia de Osakidetza. Por tanto, este abordaje integral se hace posible mediante una conjunta y estrecha intervención entre servicios sanitarios y sociales.

La tipología de patología mental es variada y extensa, siendo la que nos ocupa aquella que, por sus condicionantes, representa una mayor vulnerabilidad y gravedad. Concretamente nos referimos al trastorno mental grave (TMG en adelante), siguiendo la definición más representativa y que ha alcanzado un mayor consenso, ofrecida por el Instituto Nacional de Salud Mental de EE.UU. en 1987. Conceptualiza el TMG de manera tridimensional, por la concurrencia de tres factores que, asociados, generan una importante multiproblemática. Estas tres dimensiones son: el diagnóstico de enfermedad mental grave, fundamentalmente los trastornos psicóticos y, más en concreto la esquizofrenia; en segundo lugar, la persistencia en el tiempo, medida por la duración del tratamiento (se considera proceso crónico cuando se prolonga por un periodo superior a los dos años); y una última dimensión relacionada con su efecto en la autonomía y el proceso vital personal, es decir, la presencia de discapacidad, determinada por la existencia de una disfunción moderada o severa del funcionamiento global, medida a través del instrumento para la evaluación global del funcionamiento (GAF), que indica una afectación de moderada a severa del funcionamiento laboral, social y familiar. Este daño añadido de cronicidad y deterioro social redunda en incrementar las dificultades de inserción, siendo el aislamiento un obstáculo tal que se detecta todo un subgrupo de personas que quedan al margen de cualquier servicio de salud y, por tanto, privadas de un tratamiento psiquiátrico y rehabilitador adecuado.

Este programa nace con la intención de paliar esta peculiaridad y, por ello, está dirigido a PSH mayores de edad, con enfermedad mental grave, que viven en el municipio de Bilbao y que no están siendo atendidos por la red pública de salud mental estándar, o carecen de vínculos con ella. La necesidad de un programa asistencial específico para esta población se sustenta en la existencia de un número significativo de personas, tal como muestran las estadísticas antes mencionadas, que padecen trastornos psi- 
quiátricos y se encuentran en situación de exclusión social y de carencia de alojamiento, que actualmente viven en la calle y en recursos municipales dirigidos a esta población. Y también en que los problemas y las carestías asistenciales de esta población en muchas ocasiones no permiten, al menos inicialmente, su abordaje por los recursos asistenciales sanitarios de salud mental normalizados.

Es importante subrayar, en este sentido, que este programa no pretende ser una alternativa a las intervenciones que ofrecen los centros y servicios sanitarios de atención normalizados, sino que intenta completar el vacío asistencial en el que perviven las personas con TMG en sus estadios más funestos y desvinculados, siendo un puente facilitador y transitorio hacia la integración social completa.

\section{Antecedentes}

\subsection{Experiencias en el Estado}

En la actualidad existen equipos asistenciales sanitarios que trabajan específicamente con esta población en Madrid, Barcelona, Málaga o Canarias. En el caso de la Comunidad de Madrid, se desarrolla un programa de atención psiquiátrica en las áreas 5 y 7 de la ciudad, dependiente del Servicio de Psiquiatría del Hospital de La Paz, así como un programa de atención social a esta población (Proyecto de Apoyo y Reinserción de Personas con Enfermedad Mental Sin Hogar, PRISEMI) dependiente de la Consejería de Familia y Asuntos Sociales. En Barcelona, desde 2006 está en marcha el Programa de Salud Mental a la Población Sin Hogar de la Ciudad de Barcelona, dependiente de la Dirección de Área de Barcelona de Sant Joan de Déu-SSM y de los servicios de salud mental de la Fundación Hospital Sant Pere Claver, en colaboración con el Consorcio Sanitario de Barcelona y la Dirección de Acción Social y Ciudadanía del Ayuntamiento de Barcelona.

En 2009, el Ministerio de Sanidad, Política Social e Igualdad, a través de la Dirección General de Política Social, de las Familias y de la Infancia, decidió impulsar las actuaciones desarrolladas para abordar esta problemática de exclusión social extrema, para lo cual propuso la creación de un grupo de trabajo específico (Ministerio de Sanidad, Política Social e Igualdad, 2011).

\subsection{Experiencia en el municipio de Bilbao}

En el municipio de Bilbao se venía desarrollando desde marzo de 2006 este programa de atención a personas sin hogar que padecen trastornos mentales. El programa se gestó mediante un convenio entre la Asociación Bizitegi y el Hospital de Bermeo. La colaboración la propició la Asociación Bizitegi, que tras una experiencia acumulada de cinco años de intervención con este colectivo, alertó sobre la falta de asistencia psiquiátrica para los personas PSH con TMH que quedaban fuera de la red de atención. El objetivo era ofrecer una atención especializada orientada a crear un vínculo, mediante un tratamiento no invasivo y desarrollado en el medio del usuario.

En virtud de este acuerdo, algunos profesionales (psiquiatra, enfermería, monitores) dependientes del Hospital de Bermeo prestaban una atención específica al colectivo de PSH con TMG que carecían de asistencia. Dicho personal se desplazaba mensualmente al recurso Bizitegi y a los albergues, y atendía dos días por semana a los pacientes asignados al programa en el propio hospital de día de Begoña, en Bilbao. El programa se apoyaba para cubrir sus necesidades de ingreso psiquiátrico tanto en la Unidad de Psiquiatría del Hospital de Basurto como en las del Hospital Psiquiátrico de Bermeo.

A partir de 2010, en virtud del acuerdo interinstitucional mencionado en el apartado 1, se formaliza y amplia el programa de atención, dotándolo de recursos, clarificando el acceso a él y estableciendo los mecanismos de coordinación necesarios. Desarrollaremos a continuación estos aspectos.

\section{Descripción del programa}

Siguiendo la aplicación de los principios de rehabilitación psicosocial a las personas sin hogar con enfermedad mental, es preciso ajustarse a variables propias del colectivo, introduciendo en el abordaje las variaciones que se consideren precisas (Recalde, 2010). En este sentido, apreciamos tanto barreras internas propias del colectivo como obstáculos externos relacionados con las exigencias estructurales del contexto social, entre las que también se encuentran las causadas por la rigidez de los servicios asistenciales. La característica definitoria de este colectivo es su condición de no tener hogar, es decir, no tener un alojamiento adecuado y estable donde reorganizar la propia historia y el proyecto de vida personal asociado al aislamiento y la desvinculación social y familiar. Se considera que uno de los factores protectores de mayor envergadura es la presencia de soporte social, área en el que presentan un déficit absoluto.

A la situación de marginación propia del colectivo de PSH, se une la existente en las personas con enfermedad mental por el estigma asociado a dichos trastornos en la población general (Crespo et al., 2007; Asociación Española de Neuropsiquiatría, 2008; Muñoz et al., 2009) y que afecta a todas las personas con enfermedad mental, maximizando su efecto.

Los modelos sociológicos explican la generación de este fenómeno de sinhogarismo y los modelos psicológicos especifican la afectación individual y la particularidad de las personas susceptibles de sufrirlo. Blackhart et al. (2009) realizaron un metanálisis de 192 estudios sobre estos efectos psicológicos de la exclusión social y llegaron a la conclusión de que genera, como rasgo psíquico más potente, aplanamiento afectivo o un estado de 'neutralidad' emocional, factor altamente imposibilitador de la adherencia a los programas; ello 
apunta a que propiciar el enganche y la vinculación con el usuario resulta clave para introducir un proceso de recuperación. Es decir, resulta tan esencial ofrecerles tanto medios para vivir como motivos para ello.

\subsection{Objetivos específicos}

El programa tiene como objetivo de primer orden la captación y vinculación, a la vez que mantener unos mínimos de seguridad personal, abordando la reducción de riesgos y daños. En un segundo momento, pero inmediato al anterior, se pretende lograr la adherencia al tratamiento; el cumplimiento de un tratamiento integral, que incluya tanto el abordaje de sus necesidades más básicas como la asistencia psiquiátrica y sanitaria, y de soporte social.

Garantizar la continuidad de cuidados es una meta fundamental, pues debe evitarse que tras el alta hospitalaria se alejen del sistema de atención, ahondando de nuevo en la reincidencia hospitalaria. Habitualmente las hospitalizaciones suelen ser breves, siendo difícil en este periodo lograr una adherencia al tratamiento. Como horizonte y meta a más largo plazo nos planteamos propiciar la inserción en la red de salud normalizada.

\subsection{Metodología básica de intervención}

Encontramos que las dificultades para el abordaje sanitario y social de esta población provienen de varias vertientes:

- Dificultades propias de la patología, como la desconfianza, la escasa o nula conciencia de enfermedad, y la falta de adherencia al tratamiento. En muchos casos la demanda no parte del paciente, ni es deseada por él. Otras veces son las dificultades derivadas de la clínica propia del paciente, como la presencia de actividad productiva, la amotivación o la inestabilidad. La valoración de la presencia de consumos de drogas y su incidencia en la conducta y sintomatología presente no resulta sencilla. Es preciso un prolongado trabajo previo que permita la intervención, en coordinación con los educadores de calle.

- Desconocimiento cultural, que redunda en problemas básicos de comunicación asociados a la dificultad idiomática o expresiva, o en dificultades en filiar el carácter extraño de la conducta como patológico, o como producto de la idiosincrasia cultural.

- Problemas derivados del estilo de vida, el continuo cambio de ubicación o los hábitos en absoluto saludables desembocan en la aparición de enfermedades somáticas. Es de señalar que hay estudios que indican que la situación de sinhogarismo va asociada a una reducción en veinte años de la esperanza de vida.

- Problemas administrativos: ausencia de empadronamiento, situación irregular, ausencia de TIS; dificultades para acceder a recursos sanitarios normalizados, a recetas/medicación, a asistencia sanitaria general, a hospitalización psiquiátrica; entre otras.

- Problemas de apoyo y cobertura social: padecen alto grado de aislamiento y falta de conexiones afectivas y sociales. Se hace indispensable garantizar unos mínimos de cobertura social básica, incluido alojamiento, que permitan instaurar el tratamiento y un seguimiento básico con garantías, así como una salida viable a las posibles hospitalizaciones psiquiátricas.

Las respuestas sanitarias tradicionales resultan insuficientes para atajar los mencionados obstáculos, por lo que se instaura una metodología de acercamiento fundamentada en el modelo de tratamiento asertivo comunitario que ha venido demostrado su primacía desde hace muchos años en nuestro contexto y que tiene sus antecedentes en los programas implantados en los años setenta en EE.UU. Cumple con los requisitos que se consideran cardinales para desarrollar servicios que atiendan a esta población: el trabajo en red, la búsqueda activa (outreach) y la continuidad de cuidados centrados en la gestión de casos (case management).

Esta metodología supone una flexibilización y adaptación de las estructuras dedicadas a la asistencia de pacientes con problemas de salud mental aportando la disponibilidad de realizar intervenciones, tanto en centros como en la calle o el entorno más inmediato de la persona. Las políticas sociales de atención están alineadas con esta orientación asertiva, sustentada en la responsabilidad pública de mantener un liderazgo local y próximo en la oferta de servicios sociales de referencia. La diversidad de la demanda queda cubierta por la diferenciación de la respuesta traducida en centros y dispositivos variados en cuanto a sus prestaciones, exigencias o normas.

Otras premisas que subyacen al modelo de atención social son la búsqueda de dignificación, de la atención integral procurada desde procesos unitarios de intervención, diversificados y articulados en red. Es preciso preservar la voluntariedad como elemento primordial para desarrollar el proceso personal, reduciendo la introducción de intervenciones impositivas a las mínimas necesarias para garantizar la seguridad, la salud y las condiciones de vida más básicas. Las referencias personales estables y emotivas son esenciales para corregir experiencias traumáticas, integrar facetas personales, y comenzar y perpetuar los procesos de cambio. Además, por las características de la población diana, la reducción del riesgo y daño, así como la orientación de baja exigencia son necesariamente elementos metodológicos nucleares de este programa.

\subsection{Recursos}

\subsubsection{El equipo}

El recurso clave del programa es el equipo. Es el ancla fundamental. Dada la complejidad y singularidad del abordaje, es preciso cuidar de la cualidad 
de los equipos que trabajan con esta población. Respecto a su composición, es crucial procurar la multidisciplinariedad, que garantiza la administración de prestaciones prolijas y dispares, y que ésta se realice de manera conjugada.

La proactividad debería ser parte de la idiosincrasia del equipo. Permite aproximarse al usuario e introducirse en su medio. Conjugada con la capacidad de acompasamiento, respeta la interacción que marca el usuario, y se encamina a facilitar el empoderamiento y la responsabilidad social, sin caer en la dependencia iatrogénica, ni en la imposición. Implica una determinada actitud en la empresa de orientarse al sujeto, pues supone trasladar el contexto terapéutico habitual al medio controlado por el afectado; el encuadre grupal o individual propio de la consulta debe convertirse en un marco móvil y compartido con el resto del equipo. Superar este reto exige trasponer todas las herramientas técnicas sin contar con la contención y seguridad que aporta el entorno de atención tradicional. Este proceder requiere un importante dominio sobre la capacidad empática, la escucha activa y el prejuicio, y también habilidades de negociación y mediación que pongan de manifiesto valores como la paciencia, la tolerancia, la consideración y la aceptación de la situación del otro.

La prestación asistencial sanitaria del programa es procurada por la Red de Salud Mental de Bizkaia, dependiente de Osakidetza, utilizando como sede del programa el actual Centro de Salud de Begoña, en coordinación con las unidades de rehabilitación psiquiátricas y las unidades de psiquiatría de los hospitales generales. El equipo sanitario cuenta como recursos humanos con dos psiquiatras, una enfermera y una trabajadora social, todos ellos a tiempo parcial. Se realiza un abordaje en colaboración con los educadores de calle vinculados a servicios sociales.

El equipo social está constituido fundamentalmente por trabajadores y educadores de los programas y servicios dependientes de la Sección de Inclusión y Urgencias Sociales del Ayuntamiento de Bilbao. Intervienen principalmente el Programa de Intervención en Calle con PSH, el Servicio Municipal de Urgencias Sociales (SMUS), el Albergue Municipal de Elejabarri y el Albergue Municipal de baja exigencia.

\subsubsection{Otros recursos de apoyo}

Se cuenta también con los siguientes recursos de apoyo:

- Estancias hospitalarias: se intenta que la mayoría de los ingresos se produzcan de forma programada y tras un periodo de intervención en calle. El estado y circunstancias del usuario determinan fundamentalmente el lugar de ingreso. En el caso de pacientes en situación aguda que requieren una cobertura hospitalaria de mayor supervisión, la hospitalización inicial se realiza en la Unidad de Psiquiatría del Hospi- tal General, con el respaldo de las unidades de subagudos o rehabilitación de los hospitales psiquiátricos en el caso de necesidad de prolongar la hospitalización.

- Atención a otros problemas de salud: los problemas de salud general son atendidos, siempre que sea posible, en los servicios asistenciales normalizados correspondientes, en coordinación con los responsables del programa. En caso de dificultades derivadas de falta de empadronamiento o ausencia de TIS, por ejemplo, los pacientes son atendidos por el centro de salud correspondiente al recurso de alojamiento municipal utilizado. En ausencia de éste, la atención es procurada por el Centro de Salud de Begoña.

- Acceso a medicación: de forma excepcional, y hasta normalizar su situación, la medicación para los pacientes que carecen de TIS y de acceso a fármacos mediante emisión de recetas se provee mediante el servicio de farmacia de la Red de Salud Mental de Bizkaia, a través de la prescripción del equipo asistencial responsable del programa.

- Prestaciones de servicios sociales: el Área de Acción Social proporciona a las personas derivadas a este programa de salud mental las prestaciones propias de los servicios sociales, incluidas las necesidades básicas (alojamiento temporal, alimento, vestido), en el marco de la normativa y procedimientos vigentes.

\subsection{Componentes y fases de la intervención}

\subsubsection{Detección y derivación}

La detección de personas objeto de atención se realiza de forma ordinaria a través de los dispositivos y servicios dependientes de la Sección de Inclusión y Urgencias Sociales: Programa de Intervención en Calle con PST, Servicio Municipal de Urgencias Sociales (SMUS) y centros residenciales para PST. Cuando el Sistema de Servicios Sociales Municipales detecta una situación que puede ser objeto de este programa, deriva el Equipo de Salud Mental para Personas Sin Hogar, a través de la Jefatura de Sección de Inclusión y Urgencias Sociales, remitiéndole el documento establecido ad hoc para su valoración. Salud Mental se pone en contacto con el profesional de referencia que figura en el documento de derivación para iniciar y acordar el proceso de abordaje.

En ocasiones, la detección del caso la realiza el Servicio de Psiquiatría del Hospital, que lo derivación a la Jefatura de Sección de Inclusión y Urgencias Sociales en coordinación con el equipo de salud mental responsable del programa mediante el mismo procedimiento y encauzando las primeras intervenciones individualizadas precisas. Por su parte, los servicios sociales de base pueden proponer pacientes siempre que estén considerados en el colectivo diana propio de este programa. 


\subsubsection{Evaluación}

El paradigma de la evaluación es la alteración de la dimensión temporoespacial de la persona, tanto en su forma -pues las condiciones de dicha alteración no se dan en el ámbito habitual del consultorio profesional-, como en su contenido. Dada la peculiaridad de la intervención, resulta difícil realizar una evaluación funcional estándar. Una de las propiedades de la evaluación es la continuidad en el tiempo, y el acoplamiento al tempo que marca el usuario. Otro distintivo es que la fuente primordial de información sobre el sujeto proviene de su contexto; de lo que se observa en sus rutinas y quehaceres, de la red que tiene alrededor, del conocimiento aportado por otros profesionales que interactúan con él, o de lo rescatado de otros episodios.

Un aspecto difícil de manejar es la ocasional precariedad en la toma de decisiones, que por razones de urgencia, se debe hacer sin terminar de manera satisfactoria el proceso de evaluación. Al intervenir, carecemos de mucha información y es preciso contar con ello. También es conveniente cotejar la información que nos aporta el usuario, coordinándonos con el resto de equipos de intervención (Bravo, Recalde y Vázquez, 2010). Resulta básico escuchar las prioridades del paciente desde un interés sincero por la persona, y que ésta perciba al clínico como un aliado. En este sentido, cobra especial relevancia la exploración y el desarrollo de la motivación personal.

Como instrumento objetivo específico de evaluación, empleamos la Escala HoNos traducida por Uriarte et al. (1999) para medir todo el rango de problemas físicos, personales y sociales asociados a la enfermedad mental. Nos aporta una medida de gravedad que explora doce áreas agrupadas en problemas conductuales, clínicos y psicosociales que representamos en el Cuadro 1.

\begin{tabular}{|l|}
\hline Cuadro 1. Escala HoNOs \\
\hline Problemas conductuales \\
\hline - Conducta hiperactiva, agresiva, disruptiva o agitada. \\
- Autolesiones no accidentales. \\
- Consumo problemático de alcohol o drogas. \\
\hline Deterioro \\
\hline - Problemas cognitivos. \\
- Problemas por enfermedad física o discapacidad. \\
\hline Problemas clínicos \\
\hline - Problemas asociados a la presencia de ideas delirantes y \\
alucinaciones. \\
- Problemas en relación con el humor depresivo. \\
- Otros problemas mentales o conductuales. \\
\hline Problemas sociales \\
\hline - Problemas con las relaciones. \\
- Problemas en relación con las actividades de la vida cotidiana. \\
- Problemas con las condiciones de vida. \\
- Problemas en relación con la ocupación y las actividades. \\
\hline
\end{tabular}

Fuente: Uriarte et al. (1999).
Además de las cuestiones clínicas, psicosociales y contextuales que acabamos de mencionar resulta relevante conocer otras informaciones que habitualmente pueden pasar inadvertidas, pero que son centrales para iniciar cualquier proceso más avanzado de intervención y para valorar el pulso de su respuesta de integración. En el Cuadro 2 exponemos estos aspectos básicos del usuario que hay que recoger.

Cuadro 2. Datos e información básica que se ha de registrar

- Datos sociodemográficos.

- Datos de localización.

- Familiares o personas de contacto.

- Datos del servicio y profesional de referencia.

- Intervenciones/prestaciones.

- № derivaciones: nuevas, reinicios.

- Inicios de tratamiento.

- Abandonos de tratamiento psiquiátrico.

- Periodos de alojamiento.

- Altas a otros programas de inserción.

- Tiempo medio de permanencia en el programa.

- Tiempo medio de contacto hasta abandono.

Fuente: Elaboración propia.

\subsubsection{Planificación}

El resultado de esta exploración se plasma en el diseño progresivo del plan individualizado de atención, en el que se establecen y consensuan las necesidades detectadas, así como los objetivos de las intervenciones que se han de realizar, tanto sanitarias como sociales. Es importante no caer en tentaciones pretenciosas y crear expectativas imposibles de cumplir, ni paralizarse ante la angustia de situaciones desesperadas. El análisis funcional, racional y sistemático ayuda a introducir propósitos posibles y esperanzadores. Se realiza una gestión conjunta de cada caso por parte de Salud Mental y Acción Social del Ayuntamiento, a través de este plan conjunto, revisable periódicamente.

Los aspectos más reseñables de este plan están encaminados a encauzar y lograr, como objetivos cercanos, la estabilidad clínica, un proyecto vital que garantice la continuidad de cuidados integrado en el marco comunitario y normalizado, y, como meta final, la derivación a un programa de atención psiquiátrica estandarizado.

\subsubsection{Fases de la intervención}

La intervención no es lineal, aunque con objeto de facilitar la comprensión de la evolución, se pueden diferenciar una serie de fases. Estas fases se pueden solapar, siendo frecuentes las regresiones.

Respecto al proceso de abordaje, siguiendo la clasificación de Brandt (2003), distinguimos las siguientes etapas:

- Fase de contacto. Intervenimos a instancias de la detección realizada por los equipos de calle e 
intentamos acercarnos para conseguir un enganche. En esta fase, son aspectos primordiales el respeto temporoespacial de las coordenadas de la persona que se pretende ayudar; el acercamiento a los lugares en los que se siente segura, la flexibilidad de aceptar sus ritmos y la cautela en concederles lo que pueden tolerar como apoyo, entre otras cuestiones. Puede ser una etapa larga y laboriosa, en la que se suelen producir la mayoría de los abandonos. Se trata de generar seguridad y vencer la desconfianza.

- Fase de clarificación o diagnóstica. En esta etapa -que se va superponiendo con la siguientecomenzamos a conocer y diagnosticar a estas personas, para así poder programar un abordaje. En ocasiones las diferencias culturales, de idioma o la confusión sintomática dificultan no sólo determinar un diagnostico, sino incluso obtener la verdadera identidad de la persona.

- Fase de enganche. Se encamina a lograr una alianza terapéutica que permita el desarrollo de las intervenciones. Conlleva un proceso largo que sienta las bases a la adherencia e integración. Puede aparecer el riesgo de que se establezcan relaciones de dependencia y estancamiento.

- Fase de apoyo. Se produce la consolidación de sus resultados y persiste el soporte, aunque se haya solucionado lo que inicialmente se pretendía.

- Fase de conclusión. Supone el paso exitoso a otra dimensión. En ocasiones suele ser una fase muy larga en la que, con contactos muy escasos y espaciados, se permite la despedida, evitando recaídas.

\subsection{Coordinación y gestión de casos}

Este modelo de intervención en red es sumamente complejo y requiere de una coordinación ajustada, de llegar a acuerdos entre recursos, de trabajar objetivos comunes y de repartir tareas y responsabilidades. El equipo sanitario y social mantiene comunicación y contacto constante sobre el desarrollo de sus intervenciones. La visión compartida sobre la orientación de los casos, la dilucidación de las competencias y asignaciones de referencia en las prestaciones se van trazando a través de la reunión mensual en la que participan todos los integrantes de ambos equipos, referentes de caso incluidos.

\subsection{Supervisión y evaluación}

En un plano más global, también se requiere del análisis del funcionamiento del proyecto encaminado a garantizar la adecuación y mejora del proceso. De ello se encargan las personas responsables del programa de los dispositivos de Salud Mental y Acción Social, a través de reuniones semestrales que tienen por objeto evaluar el funcionamiento del programa, así como plantear las correspondientes propuestas de mejora. Toda esta actividad queda reflejada en una memoria anual conjunta.
Esa valoración se fundamenta en el análisis de los indicadores mostrados en el Cuadro 3. Los indicadores son un instrumento de medición cuantitativo que permiten evaluar y controlar el proceso de manera objetiva. Los indicadores que hemos planteado son muy pocos, pero reflejan de manera clara y sencilla la realidad que pretenden medir. Son sensibles, pues permiten apreciar el resultado de los cambios, y analizar las tendencias y la evolución. Resultan útiles para establecer estándares de referencia.

Los indicadores pueden agruparse por el campo al que se refieren. Un bloque se centra en las peculiaridades más significativas del perfil de atención, lo que nos descubre de manera más fehaciente las características específicas de nuestro colectivo, sus variaciones, y facilita poder cotejarlas con lo que aparece en la bibliografía existente sobre otras experiencias. Contemplar aspectos tan básicos como el sexo -dada la triple vulnerabilidad que sufren las mujeres-, la edad de los usuarios, su procedencia, la incidencia de casos nuevos, los reinicios o el tipo de patología que padecen -reflejada en su diagnóstico psiquiátrico- permite conocer la dimensión de la problemática, lo cual, unido a las variaciones evolutivas que van apreciándose, ayuda a establecer unos criterios de estratificación elementales y a prever tendencias futuras de necesidad.

El segundo conjunto de indicadores se focaliza en lo que son los resultados asistenciales, y nos aporta información sobre el cumplimiento de objetivos del proyecto y su eficacia. Las tasas de adherencia y abandono nos hablan de la consecución de vinculación; los ingresos hospitalarios, de la gravedad y la necesidad de intervenciones de alta intensidad; las derivaciones sociosanitarias sugieren el grado de integración social. En definitiva, estos indicadores reflejan el grado de éxito que tienen nuestras intervenciones de acuerdo a las expectativas planteadas, miden la eficacia del programa y hacen posible aprender y establecer acciones de mejora.

\begin{tabular}{|l|}
\hline Cuadro 3. Indicadores de actividad y resultados \\
\hline Indicadores de perfil \\
\hline - Aspectos sociales y demográficos: \\
- Edad. \\
- Sexo. \\
- Procedencia. \\
- Diagnóstico psiquiátrico. \\
- Incidencia, casos nuevos. \\
\hline Indicadores de resultados \\
\hline - Abandonos. \\
- Reinicios. \\
- № de casos en tratamiento comunitario en el programa. \\
- Hospitalizaciones psiquiátricas. \\
- Altas a otros programas, incluyendo servicios de salud mental \\
estándar. \\
- Tiempo medio de permanencia en el programa. \\
- Tiempo medio de contacto hasta abandono.
\end{tabular}

Fuente: Elaboración propia. 


\section{Resultados}

Los indicadores asistenciales anteriormente expuestos nos aportan información relevante para valorar el desarrollo del programa. La trayectoria de varios años de experiencia conforma una tendencia estable, que da consistencia y seguridad a la interpretación de los datos. Seguidamente analizaremos los datos del programa del periodo comprendido entre marzo de 2006 y septiembre de 2012.

Desde el inicio del programa, se han tratado más de 90 casos. Respecto al sexo, la mayoría han sido hombres (82\%), y aquí coincidimos con Cabrera y Rubio (2003), que calculan que el número de mujeres dentro del colectivo de personas sin hogar representa en torno al $18-20 \%$, por lo que hablaríamos de porcentajes similares a los existentes en Bilbao. La edad media de las personas atendidas ha sido de 41 años, pero con una gran desviación típica, lo que muestra segmentos de edad muy diferenciados de usuarios muy jóvenes y personas con edad muy avanzada. En relación a su procedencia, el $54 \%$ ha sido población inmigrante, alrededor de un $20 \%$ han sido vizcaínos, y el resto, de otras comunidades del Estado. Además, en los dos últimos años se aprecia un incremento de la población inmigrante. En cuanto a su perfil psiquiátrico, el trastorno mayoritario corresponde al espectro de la psicosis (70\%), siendo el diagnóstico fundamental el de esquizofrenia. Estos datos son similares a los aportados por la literatura sobre el tema.

Tabla 1. Indicadores de perfil (\%)

\begin{tabular}{l|c|c|c|c|c|c|c}
\hline Indicadores & $\mathbf{2 0 0 6}$ & $\mathbf{2 0 0 7}$ & $\mathbf{2 0 0 8}$ & $\mathbf{2 0 0 9}$ & $\mathbf{2 0 1 0}$ & $\mathbf{2 0 1 1}$ & 2012* \\
\hline $\begin{array}{l}\text { Mujeres en el } \\
\text { programa }\end{array}$ & 25 & 0 & 10 & 21 & 18 & 17 & 22 \\
\hline $\begin{array}{l}\text { Diagnóstico } \\
\text { de } \\
\text { esquizofrenia } \\
\text { y otras } \\
\text { psicosis }\end{array}$ & 100 & 100 & 100 & 62 & 51 & 59 & 71 \\
\hline $\begin{array}{l}\text { Colectivo } \\
\text { inmigrante }\end{array}$ & 38 & 40 & 30 & 40 & 53 & 57 & 59 \\
\hline
\end{tabular}

*Datos correspondientes a septiembre de 2012.

Fuente: Elaboración propia.

En el análisis secuencial, se observa una tendencia al incremento en esta población de jóvenes con patología dual, inmigrantes y un aumento de mujeres. Estas últimas suelen padecer una patología más grave y una historia de abusos físicos y sexuales que se mantiene durante la propia situación de sinhogarismo.

Tabla 2. Indicadores de resultado (\%)

\begin{tabular}{l|c|c|c|c|c|c|c}
\hline Indicadores & $\mathbf{2 0 0 6}$ & $\mathbf{2 0 0 7}$ & $\mathbf{2 0 0 8}$ & $\mathbf{2 0 0 9}$ & $\mathbf{2 0 1 0}$ & $\mathbf{2 0 1 1}$ & 2012* \\
\hline Casos nuevos & 100 & 45 & 77 & 45 & 57 & 35 & 35 \\
\hline Abandonos & 25 & 45 & 0 & 5 & 14 & 11 & 10 \\
\hline $\begin{array}{l}\text { Derivados a } \\
\text { programas } \\
\begin{array}{l}\text { sanitarios/ } \\
\text { sociosanitarios }\end{array}\end{array}$ & 0 & 0 & 0 & 5 & 6 & 22 & 10 \\
\hline
\end{tabular}

*Datos correspondientes a septiembre de 2012.

Fuente: Elaboración propia.
En relación a los resultados asistenciales, se ha conseguido una adherencia media del $75 \%$. Las altas exitosas han sido casi similares al porcentaje de abandonos. Aproximadamente un tercio de ellas corresponden a retornos a su lugar de procedencia (27\%), y otro tercio han sido motivadas por derivación a otros programas tras lograr su estabilidad y vinculación. Se mantiene el abordaje de más de 30 personas.

Los resultados sobre la evolución de la asistencia permiten comprobar que la tasa de abandonos se ha ido reduciendo: era mayor en los primeros años del programa y actualmente es del $10 \%$. Asimismo se aprecia que al inicio del programa, como era de esperar, abundaron los casos nuevos, pero esta tendencia se ha estabilizado los dos últimos años. La adherencia al programa, así como la derivación a otros servicios, mantienen una tendencia positiva estable.

\section{Conclusiones}

En primer lugar, señalamos las insuficiencias más inmediatas de este colectivo. A las carencias primordiales y más específicas de sustento básico -comida, alojamiento, higiene o vestido-, añadimos las necesidades de atención psiquiátrica y orgánica que estas personas padecen por estar aquejadas de una enfermedad mental grave. Esta situación se hace más compleja por las dificultades administrativas, burocráticas y legales derivadas de las irregularidades en documentación, la precariedad económica y administrativa, y la presencia de demandas judiciales, e incluso penales, que frecuentemente van asociadas a estas circunstancias de exclusión social. Su situación socioafectiva queda afectada por la marcada ausencia de soporte familiar o social, y la traumática ruptura biográfica.

En segundo lugar, la experiencia abierta de intervención en estos seis años nos ha aportado información sobre las características de la muestra y su peso como factores de diferenciación y determinación de comportamientos. Esta exploración nos lleva a apreciar una cierta segmentación en este colectivo. Se encuentran diferentes subgrupos que requieren de complejas soluciones:

- Mujeres con un importante grado de deterioro físico y social, y con riesgo importante de ser víctimas de violencia de género; en definitiva, con una triple vulnerabilidad: carecer de hogar, tener una enfermedad mental grave y ser mujer. Es un colectivo minoritario, pero que presenta una problemática abigarrada y de graves consecuencias. Suelen tener un mayor deterioro y desestructuración que los hombres. Presentan multiproblemática orgánica, con más frecuencia por la acumulación de cinco o más trastornos de salud. Padecen mayor número de enfermedades de transmisión sexual, problemas circulatorios, óseos y musculares. El consumo de tóxicos les genera consecuencias psicosociales y orgánicas más graves, por su mayor sensibilidad biometabólica. Terminan en 
una situación de sinhogarismo por motivos diferentes, y las complicaciones familiares y sociales derivadas de ello son mayores en lo que afectan a terceros (embarazos, menores). Una parte importante de las mujeres sin hogar son víctimas de violencia de género por parte de desconocidos, o incluso de sus parejas o de otros sujetos cercanos de su red (Ramiro, 2010). Las redes sociales naturales y los servicios sociales se activan con más intensidad cuando detectan este estado de vulnerabilidad en las mujeres.

- Jóvenes inmigrantes con importantes problemas psicopatológicos unidos a consumo de tóxicos (cannabis, disolventes). Población más joven con trastornos por consumo de tóxicos, con un incremento de trastornos de personalidad graves.

- Personas con una trayectoria biográfica de sinhogarismo refractaria a la integración social y, simultáneamente, una enfermedad mental de larga data. Se trata de una población más anciana y crónica, en la que prevalecen los trastornos de tipo psicótico, frecuentemente asociados al abuso de alcohol, que conlleva mayor patología física y demencias, entre otros daños.

En tercer lugar, se demuestra la eficacia del programa por sus resultados asistenciales. La elevada adherencia, constatada en el incremento del cumplimiento de tratamiento psiquiátrico, la adhesión farmacológica, y la participación en consulta psiquiátrica y en cuidados de enfermería. Fundamentalmente se estabilizan y mantienen en tratamiento las personas que tienen un diagnóstico del espectro de la esquizofrenia. También se advierte éxitos terapéuticos en las mujeres tras intervenciones complejas y costosas.

La tasa de abandonos es baja, e incluso ha disminuido ligeramente. También se comienza a apreciar entre las personas que abandonan el programa tras estancias cortas, las variables comunes de ser varón, joven, con diagnóstico de trastorno de personalidad asociado con consumo de tóxicos. Una de las razones que propicia la desaparición del programa y del medio es la presencia de cargos judiciales o penales.

Se ha incrementado la atención a las personas diagnosticadas de esquizofrenia y otras psicosis. Creemos que los profesionales que trabajan en primera línea de cribado han desarrollado la sensibilidad en la detección y el manejo de los trastornos de salud mental grave, lo que ha permitido una acertada adecuación del recurso.

La gravedad psicopatológica de los casos, la ausencia total de soporte social y la carencia de recursos de sostén en el alojamiento condicionan que las hospitalizaciones sean elevadas, tanto en los ingresos breves realizados en las unidades de psiquiatría de hospitales como los que requieren de estancias más prolongadas en las unidades de rehabilitación de los hospitales psiquiátricos. La situación de la persona afectada suele ser en ocasiones tan extrema que el primer contacto con el ámbito sanitario es a través del ingreso psiquiátrico, siendo ésta la puerta de entrada al programa. La concentración de personas con una enfermedad mental grave que tienen como característica fundamental su desvinculación con la red de salud mental estándar podría explicar esta necesidad de utilizar un recurso de alta contención.

Planteado inicialmente para unas 35 a 40 plazas, podríamos considerar que el programa se ajusta a la necesidad de la población de referencia. No hay lista de espera y no se produce saturación, al menos en el ámbito comunitario; ni siquiera hay un incremento lineal de la necesidad, pues el número de casos nuevos permanece estable. Se mantienen en el programa más de 30 personas. Por otro lado, se aprecia un alto movimiento y renovación de personas en el programa (25-30\%, aproximadamente), coherente con un porcentaje también elevado de altas. Es de reseñar que la antigüedad del programa incide en que comiencen a producirse reinicios. Respecto al motivo de las altas, analizadas en sentido absoluto, el porcentaje de abandonos es similar al que se produce por incorporación a un recurso sociosanitario más normalizado y se aproxima al porcentaje de usuarios que regresan a su lugar de procedencia.

Podemos decir que el análisis de la evolución de la asistencia y los resultados arriba expuestos avalan la eficacia del programa: altas tasas de adherencia al programa con escasos abandonos. Sin embargo, es preciso conseguir más derivaciones a los servicios de asistencia de salud mental y de asistencia social normalizados. Quedaría pendiente un estudio más pormenorizado sobre los factores que dificultan este desarrollo.

Respecto a las dificultades encontradas, los mayores obstáculos provienen del aislamiento, la patología y las resistencias del propio colectivo. La escasez de recursos de vivienda protegida, tanto en número como variedad, y la lentitud de los aspectos burocráticos y administrativos son barreras especialmente obstaculizadoras. También encontramos los anteriormente mencionados problemas de desvinculación del programa.

Examinando la realidad de los equipos de atención, encontramos complicaciones producto de la complejidad de conjugar la visión sobre los casos derivada de los distintos enfoques disciplinarios sanitario y social, diferencias que pueden limarse a través de encuentros formativos comunes. Por otro lado, los inconvenientes generados por la propia dinámica metodológica de movilidad y actuación inmediata se van subsanando, introduciendo estrategias que orientan a definir mejor las competencias de intervención y mediante la referencia en la gestión de los casos.

A modo de reflexión última, diríamos que identificamos dos factores de éxito fundamentales. Uno es el cambio en la metodología asistencial: es necesario cambiar el sentido estructural del manejo del espacio y del tiempo; es preciso dirigirse al lugar en el que está la persona, mostrarse disponible y flexible con los ritmos, con una metodología asertiva, pero de 
acercamiento no intimidatorio. Las expectativas de baja exigencia, la tolerancia, la disponibilidad y la paciencia son valores de primer orden. El segundo factor de éxito es la coordinación entre los ámbitos sociales y sanitarios, tanto a nivel macroinstitucional, por la creación y apoyo en el mantenimiento del programa, como a nivel microinstitucional, en lo correspondiente a los equipos que prestan la asistencia directa, los cuales, unidos y focalizados en una orientación conjunta hacia la resolución de los casos compartidos, favorecen la vinculación de los usuarios hacia una vida normalizada, la adherencia al tratamiento y el inicio de un camino hacia la reconstrucción de un proyecto personal. 
AIERDI, X. et al. (2009): Perfil y necesidades de las personas sin hogar en Bizkaia, Bilbao, Diputación Foral de Bizkaia [<http://www.bizkaia.net/Home2/ Archivos/DPTO3/Temas/Pdf/sintecho_final_ opt_cas.pdf〉].

ÁREA DE ACCIÓN SOCIAL (2012): Memoria 2011 Txostena, Bilbao, Ayuntamiento de Bilbao [rhttp://goo. $\mathrm{gl} / 1 \mathrm{fPBV}\rangle]$.

ASOCIACION ESPAÑOLA DE NEUROSPIQUIATRIA (2008). Documento de consenso sobre la promoción de la salud mental, prevención de la salud mental y disminución del estigma. Revista de la Asociación Española de Neuropsiquiatría, 103. [〈http://www.aen.es/docs/CTecnic08.pdf〉].

BLACKHART, G. C. et al. (2009): “Rejection elicits emotional reactions but neither causes immediate distress nor lowers self-esteem: A meta-analityc review of 192 studies on social exclusion", Personality and Social Psychology Review, no-13, págs. 269-309.

BRANDT, P. (2003): "Encuentro con los enfermos mentales que viven en la calle", Cuadernos de Psiquiatría Comunitaria, vol. 3, nํㅡ 2, págs. 148-157.

BRAVO, M. F.; RECALDE, F.; y VÁZQUEZ, M. I. (2010): "Evaluación de personas sin hogar con enfermedad mental”, Asociación Madrileña de Rehabilitación Psicosocial, $\mathrm{n} \div \mathbf{2 5}$, págs. 16-25 [<http://www.amrp.info/Boletines/ Boletin25-2010.pdf $>$.

CABRERA P.; y RUBIO, M. J. (2003): Personas sin techo en Madrid. Diagnóstico y propuestas de actuación, Madrid, Universidad Pontificia Comillas.

CRESPO, M. et al. (2007): "Stigma associated with severe and persistent mental illness among the general population of Madrid (Spain): Descriptive study", Community Mental Health Journal, no 44, págs. 393-403.
EUSTAT (2007): Encuesta sobre las Personas sin Hogar. Análisis de resultados. 2005, Vitoria-Gasteiz, Eustat [http://www.eustat.es/elementos/ eleo003600/ti_Encuesta_sobre_las_ Personas_sin_Hogar_Analisis_de_resultados/ infooo3600_c.pdf].

FAZEL, S. et al. (2008): “The prevalence of mental disorders among the homeless in Western countries: Systematic review and meta-regression analysis", PLoS Medicine, vol. 5, $\mathrm{n}$ - 12, e225 [khttp://dx.doi.org/10.1371/journal. pmed.0050225>].

MINISTERIO DE SANIDAD, POLÍTICA SOCIAL E IGUALDAD (2011): Configuración de una red local de atención a personas sin hogar integrada en el Sistema Público de Servicios Sociales. Cien argumentos y propuestas, serie Informe, Estudios e Investigación 2011, Madrid, Ministerio de Sanidad, Política Social e Igualdad [<http://www.msps.es/gl/novedades/ docs/10oargumentosEspayol.pdf>].

MUÑOZ, M. (2010): "Qué sabemos y qué necesitamos saber sobre la salud mental de PSH", Asociación Madrileña de Rehabilitación Psicosocial, $\mathrm{n}$ 025, págs. 9-15 [<http://www.amrp.info/Boletines/ Boletin25-2010.pdfs].

MUÑOZ, M.; VÁZQUEZ, C.; y CRUZADO, J. A. (2005): Personas sin hogar en Madrid. Informe psicosocial y epidemiológico, Madrid, Comunidad Autónoma de Madrid.

NATIONAL INSTITUTE OF MENTAL HEALTH (1987): Towards $a$ Model for a Comprehensive Community Based Mental Health System, Washington DC, National Institute of Mental Health.

RAMIRO M. S.; IRAETA B. (2010): “Nuevos retos en la intervención con personas sin hogar con 
enfermedad mental”, Asociación Madrileña de Rehabilitación Psicosocial, $\mathrm{n} \div \mathbf{0} 25$,

págs. 9-15 [<http://www.amrp.info/Boletines/ Boletin25-2010.pdf)].

RECALDE, F. (2010): "Rehabilitación psicosocial con personas sin hogar con enfermedad mental", Asociación Madrileña de Rehabilitación Psicosocial, no 25, págs. 30-34 [rhttp://www. amrp.info/Boletines/Boletin25-2010.pdf〉].
URIARTE, J. J. et al. (1999): “Presentación de la traducción al castellano de la escala HoNOS (Health of the Nation Outcome Scales)", Psiquiatría Pública, vol. 11, nํㅜ 4, págs. 93-101.

VÁZQUEZ, M. I. (2010): “Atención psiquiátrica a personas sin hogar que padecen enfermedad mental grave", Asociación Madrileña de Rehabilitación Psicosocial, no 25, págs. 35-42 [rhttp://www. amrp.info/Boletines/Boletin25-2010.pdf〉]. 